Complexity and composition of synthesized web services

Non Peer-reviewed author version

Fan, Wenfei; GEERTS, Floris; GELADE, Wouter; NEVEN, Frank \& Poggi, Antonella (2008) Complexity and composition of synthesized web services. In: Lenzerini, Maurizio \& Lembo, Domenico (Ed.) Proceedings of the Twenty-Seventh ACM SIGMOD-SIGACT-SIGART Symposium on Principles of Database Systems. p. 231-240..

Handle: http://hdl.handle.net/1942/8497 


\section{Complexity and Composition of Synthesized Web Services}

\author{
Wenfei Fan \\ University of Edinburgh \& \\ Bell Labs \\ wenfei@inf.ed.ac.uk
}

\author{
Floris Geerts \\ University of Edinburgh \\ fgeerts@inf.ed.ac.uk
}

\author{
Wouter Gelade \\ Hasselt University \& \\ Transnational Univ. of Limburg \\ wouter.gelade@uhasselt.be
}

\author{
Frank Neven \\ Hasselt University \& \\ Transnational Univ. of Limburg \\ frank.neven@uhasselt.be
}

\author{
Antonella Poggi \\ Sapienza Università di Roma \\ poggi@dis.uniroma1.it
}

\begin{abstract}
The paper investigates fundamental decision problems and composition synthesis for Web services commonly found in practice. We propose a notion of synthesized Web services (SWS's) to specify the behaviors of the services. Upon receiving a sequence of input messages, an SWS issues multiple queries to a database and generates actions, in parallel; it produces external messages and database updates by synthesizing the actions parallelly generated. In contrast to previous models for Web services, SWS's advocate parallel processing and (deterministic) synthesis of actions. We classify SWS's based on what queries an SWS can issue, how the synthesis of actions is expressed, and whether unbounded input sequences are allowed in a single interaction session. We show that the behaviors of Web services supported by various prior models, data-driven or not, can be specified by different SWS classes. For each of these classes we study the non-emptiness, validation and equivalence problems, and establish matching upper and lower bounds on these problems. We also provide complexity bounds on composition synthesis for these SWS classes, identifying decidable cases.

Categories and Subject Descriptors: H.3.5 [Information Storage and Retrieval]: Online Information Services - Web-based services; F.4.1 [Mathematical Logic and Formal Languages]: Mathematical Logic - Computational Logic
\end{abstract}

General Terms: Languages, Theory, Design, Verification.

\section{Introduction}

The prevalent use of Web services highlights the need for studying fundamental decision problems associated with Web services. Given a service and a transaction, one wants to know whether the transaction is the result of a run of the service. This problem is referred to as the validation prob-

Permission to make digital or hard copies of all or part of this work for personal or classroom use is granted without fee provided that copies are not made or distributed for profit or commercial advantage and that copies bear this notice and the full citation on the first page. To copy otherwise, to republish, to post on servers or to redistribute to lists, requires prior specific permission and/or a fee.

PODS'08, June 9-12, 2008, Vancouver, BC, Canada.

Copyright 2008 ACM 978-1-60558-108-8/08/06 ...\$5.00. lem $[2,29]$ and is useful for, e.g., fraud detection. We also want to determine whether two given services are equivalent (the equivalence problem $[2,29]$ ). This allows us to replace a service with an equivalent yet cheaper one. We are also interested in finding out, at compile time, whether a service makes sense, i.e., it can generate valid transactions (the nonemptiness problem). These decision problems are not only of theoretical interest, but are also important in practice.

Another central technical issue concerns Web service composition. When a client request cannot be satisfied by any available service, one wants to automatically generate a mediator [7] to coordinate available services and meet the client's requirements. The composition synthesis problem is to determine, given a request and a set of available services, whether there exists such a mediator that, taken together with the available services, delivers the requested service.

The complexity of the decision problems and composition synthesis highly depends on how Web services are defined. A variety of standards (e.g., [7, 25, 27, 30, 31]) and models (e.g., $[2,5,6,12,13,15,18,26,29])$ have been proposed for Web services (see [21] for a survey). Some of the models specify the behaviors and interactions of Web services in terms of finite-state automata (FSA) [6, 15, 18]. Other models are based on data-driven transducers that support (infinite) states parametrized with input relations and local databases, and at each state, generate actions via queries on the data $[2,5,12,13,29]$. The validation and equivalence problems, as well as other interesting verification problems, have been studied for the transducers [2, 12, 13, 29]. While composition synthesis was studied for FSA abstractions [6, $18,24]$, little is known for data-driven transducers.

To study the decision problems and composition synthesis for various models in a comparative basis, this paper proposes a notion of synthesized Web services (SWS's) as a uniform formalism to characterize FSA and transducer abstractions. An SWS specifies the behavior of a service in terms of actions, i.e., external messages and database updates, generated in response to various sequences of input messages. As opposed to prior models, SWS's advocate deterministic synthesis of actions and parallel processing.

Example 1.1: Consider a service for booking travel packages to Disney World Orlando. Customers commit to purchase a package only if they can get (1) a reasonable airfare, 


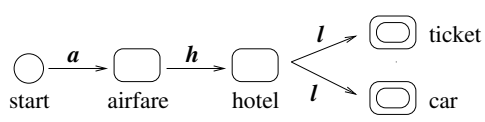

(a) An FSA expressing the service for travel packages

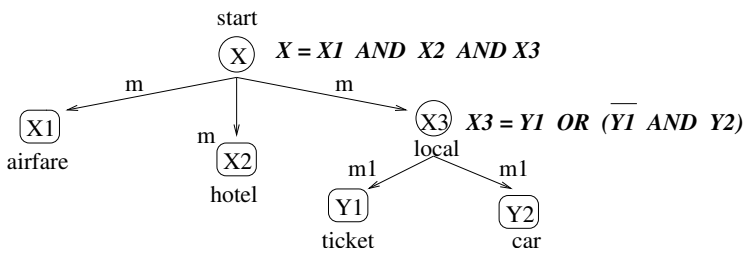

(b) SWS specification

Figure 1: FSA specification vs. SWS specification

(2) a nice hotel, and (3) either (a) Disney tickets or (b) a rental car with discount. An FSA abstraction of the service is shown in Fig. 1(a), in which airfare, hotel, ticket and car denote FSA specifications for booking flights, hotel, tickets and rental car, respectively (details omitted). Similarly a datadriven transducer can be specified, which outputs actions at each state transition. Such specifications are, however, hampered by the following concerns. First, there is no temporal dependency between airfare and hotel checking; they can be conducted in parallel, instead of sequentially. Second, the customers make a commitment only if conditions 1-3 are conjunctively satisfied. Thus the booking of flight and hotel should be deferred until condition 3 is inspected, since the failure of 3 may force the earlier transactions to rollback. Third, although conditions (a) and (b) are disjunctive, the customers may want to deterministically commit to one of the two options, rather than make decisions nondeterministically or worse, commit to book both rental car and tickets.

An SWS specification is depicted in Fig. 1(b), along the same lines as alternating finite automata (AFA). Upon receiving an input message $m$ from a user (e.g., travel date and price range), the SWS checks airfare, hotel, followed by car rental and ticket sale based on input message $m_{1}$ specifying user requirements, in parallel. Each state inspects a certain condition and keeps its truth value in a Boolean variable. The value of the variable may also be computed by a Boolean formula on variables associated with the successor states. For example, $X_{3}$ is defined by $Y_{1} \vee\left(\bar{Y}_{1} \wedge Y_{2}\right)$, in favor of ticket sale when both $Y_{1}$ and $Y_{2}$ are true, and it is evaluated as soon as the values of $Y_{1}, Y_{2}$ are available. The SWS returns true only if the condition at the start state holds.

A data-driven SWS can be specified similarly. At each state certain actions can be generated, beyond just Boolean values, via queries on the input messages and databases (e.g., information about flight tickets and hotel rooms). Actions are passed upward and synthesized at each state. The actions are generated deterministically, and are not committed until the condition at the start state is confirmed to hold. With SWS's one can specify various versions of the service, data-driven or not, in a uniform framework.

These SWS's advocate parallelism by decoupling unnecessary temporal dependencies, and support "backward" determinism to synthesize actions (messages and updates). $\quad \square$

In a nutshell, an SWS specifies the observable behavior of a Web service in response to inputs from users or other services. It is defined with a set of states parametrized with messages and a local database. At each state it simultaneously issues multiple queries to the database and input message, and passes the result of each query to a successor state.
It generates actions when no more successor states can be triggered. The actions are passed upward to its parent state, which synthesizes actions from its successor states, again via a query. The actions produced at the start state are the output of the SWS. Intuitively, for each input sequence, the output indicates the behavior of the service in a communication session. The sessions are "flexible" in that they are determined by the lengths of the input sequences. At the end of each session the actions are committed, i.e., the external messages are sent and the updates are executed on the local database. The behavior of the Web service is thus captured by the runs of the SWS over all possible input sequences.

Main results. We characterize various service models with different classes of SWS's. For each of the classes we establish matching lower and upper bounds on the decision problems, and provide complexity on its composition synthesis.

FSA and transducer abstractions. We denote various classes of SWS's by $\operatorname{SWS}\left(\mathcal{L}_{\mathrm{Msg}}, \mathcal{L}_{\mathrm{Act}}\right)$, where $\mathcal{L}_{\mathrm{Msg}}$ and $\mathcal{L}_{\text {Act }}$ are the languages in which transition queries and action synthesis are expressed, respectively. We consider $\mathcal{L}_{\mathrm{Msg}}$ and $\mathcal{L}_{\text {Act }}$ ranging over propositional logic (PL), conjunctive queries (CQ), union of conjunctive queries (UCQ) and firstorder logic (FO). For each class we also study its subclass $\operatorname{SWS}_{\mathrm{nr}}\left(\mathcal{L}_{\mathrm{Msg}}, \mathcal{L}_{\mathrm{Act}}\right)$ consisting of SWS's that are not recursively defined,i.e., for services that take a bounded number of input messages in a communication session. We examine Web services defined via FSA or transducer abstractions, and show that their behaviors can be specified by various SWS classes. We show that FSA of the Roman model [6] can be expressed in SWS(PL, PL), while data-driven transducers of $[2,12,13,29]$ can be expressed in SWS(FO, FO).

Decision problems. We study the non-emptiness, validation and equivalence problems for various $\operatorname{SWS}\left(\mathcal{L}_{\mathrm{Msg}}, \mathcal{L}_{\text {Act }}\right)$ and their nonrecursive subclasses $\operatorname{SWS}_{\mathrm{nr}}\left(\mathcal{L}_{\mathrm{Msg}}, \mathcal{L}_{\text {Act }}\right)$. We establish lower and upper bounds on these decision problems, all matching, ranging from NP-complete to undecidable. The results are proved using a variety of techniques, including finite model constructions and a wide range of reductions.

Composition synthesis. We also provide complexity bounds

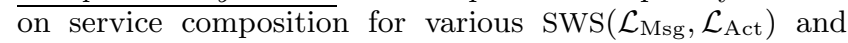
$\operatorname{SWS}_{\mathrm{nr}}\left(\mathcal{L}_{\mathrm{Msg}}, \mathcal{L}_{\mathrm{Act}}\right)$ classes. The results are established by, among other things, exploring connections between composition synthesis and (equivalent) query rewriting using views $[3,8,14,23]$. We show that composition synthesis is infeasible in most of the cases. In light of these undecidability results we identify several decidable cases.

To our knowledge, this work is among the first efforts to study the complexity of composition synthesis for datadriven Web services. Moreover, the results on the decision problems provide a comprehensive picture of the complexity of static analyses for Web services with action synthesis. The notion of SWS's allows us to study various abstractions of Web services in a uniform model. In addition, its capability of expressing synthesis of actions is a step toward specifying practical Web services with aggregations, such as finding a travel package with minimum cost.

Related work. There have been a number of standards for specifying Web services, e.g.,WSDL [31], WSCL [30], OWLS [25], SWFL [27], BEPL [7]. A variety of models have also been developed to characterize services supported by those standards, e.g., [2, 5, 6, 12, 13, 15, 18, 26, 29]. The Roman model [6] specifies a Web service as a deterministic finite 
state automaton (DFA). This model is not data-driven: neither queries nor database updates are considered. A notion of guarded automata [15], an extension of (nondeterministic) Mealy machine, is proposed to characterize conversation protocols. Extending the two, Colombo [5] models a service via a guarded nondeterministic finite state automaton (NFA), and incorporates world states of local databases. The model of [26] extends an NFA by directing transitions with input and output. Data-driven transducer abstractions are introduced in [2] and extended in [12, 13, 29]. Notably is the powerful model of $[12,13]$. It specifies transitions and generates actions via FO queries, and supports infinite states as well as other features such as asynchronous message passing and ideal vs. lossy communication channels, which are not considered in this paper. As remarked earlier, SWS's aim to characterize FSA and transducer abstractions in a uniform framework, and emphasize synthesis of actions generated by services. To our knowledge, except the split-join operator of OWL-S [25], action synthesis does not find counterparts in FSA or transducer models. The connection between these models and SWS's will be explored in Section 3.

A variety of verification problems specified in terms of e.g., linear-time temporal first-order logic, have been studied for transducer abstractions of data-driven services [2, $12,13,29]$. In particular, $(\log )$ equivalence and validation problems are shown undecidable for transducers defined in terms of FO queries, but they become decidable with certain restrictions, e.g., fixed database, bounded inputs, or decidable FO fragments [2, 29]. Validation problems have also been studied for conversation protocols $[15,16]$. We revisit equivalence and validation analyses for SWS's in this paper.

While there has been a host of work on Web service composition, few complexity bounds are known. An Expтime upper bound is established in [6] on composition synthesis of services defined in the Roman model, by reduction to the satisfiability problem for propositional dynamic logic (PDL). A matching lower bound is given for the Roman model in [24]. The upper bound is proved to hold for an extension of the Roman model with lookahead operations [18]. To our knowledge, the only complexity results on composition synthesis for data-driven services are 2ExPTIME upper bounds given in [5] for special cases of services definable in Colombo, also by reduction to decision problems for PDL.

Another issue for service composition concerns orchestration [21]. It is to determine whether available services can be coordinated, without building a mediator, such that when taken together, these services deliver a requested service. We focus on composition synthesis only in this paper.

There is a close connection between SWS composition synthesis of Web services and query rewriting using views. This connection will be studied in Section 5 .

Organization. Section 2 defines SWS's. Section 3 characterizes FSA and transducer abstractions with various classes of SWS's. For each of these classes, Sections 4 and 5 study the decision problems and the composition synthesis problem, respectively. Section 6 summarizes the main results and identifies open problems.

\section{Synthesized Web Services}

In this section we define synthesized Web services (SWS's).

An overview. Given a sequence $\mathcal{I}=I_{1}, \ldots, I_{n}$ of input messages, an SWS $\tau$ iteratively issues queries to $\mathcal{I}$ and a local database $D$, and produces an output relation $\mathcal{O}$ denoting actions, i.e., tuples to be inserted into or deleted from $D$, and external messages to be sent to other services or users.

The run of the SWS $\tau$ on $\mathcal{I}$ can be illustrated using an execution tree of depth at most $n+1$. The tree is built topdown, starting from the root. Consider the tree of depth $j \leq n$ constructed so far. At each leaf node $v$ of the tree, $\tau$ spawns $k$ children of $v$, in parallel, following a transition rule; at each child $u_{i}$ it issues a query to $D$ and $I_{j}$, and stores the result in a message register $\operatorname{Msg}\left(u_{i}\right)$. Intuitively, $\tau$ processes input $I_{j}$ at the $j$-th level of the tree. The expansion of the tree proceeds at $u_{i}$ except when (a) $j=n+1$ (i.e., all inputs have been processed), or (b) $\operatorname{Msg}\left(u_{i}\right)=\emptyset$ (i.e., the input $I_{j}$ is not meaningful), or (c) no further expansion is required by the transition rule (i.e., $\tau$ reaches a "final" state). If any of the conditions are satisfied, actions are generated at $u_{i}$, via a query to $D, I_{j}$ and $\operatorname{Msg}\left(u_{i}\right)$, and are stored in an action register $\operatorname{Act}\left(u_{i}\right)$. The parent $v$ of $u_{i}$ generates its own actions via a query on the action registers $\operatorname{Act}\left(u_{i}\right)$ of all of its children, following a synthesis rule. The process continues until the actions at the root are generated, which are returned as the output $\mathcal{O}$ of the run of $\tau$ on $\mathcal{I}$ and $D$.

In a nutshell, $\mathcal{O}$ denotes the actions of $\tau$ in response to a sequence $\mathcal{I}$ of input messages in a communication session. The notion of sessions is proposed in [12], denoting runs beginning at login and ending at logout such that no database updates occur within a session. Here we adopt a more flexible notion: a session is determined by an input sequence $\mathcal{I}$, and the actions of $\tau$ are committed at the end of the session. This notion of sessions suffices for the study of the decision problems and composition synthesis of SWS's, for which we consider the behaviors of an SWS in response to all possible input sequences. One can also treat a long (possibly infinite) input sequence as a list of consecutive sessions, by adding a delimiter \# to indicate the end of a session, such that actions are committed whenever \# is encountered.

Notations. An SWS $\tau$ is defined on a relational schema $\mathcal{R}$ for local databases $D$, an input schema $R_{\text {in }}$ for input messages $\mathcal{I}$, and an external schema $R_{\text {out }}$ for output relations $\mathcal{O}$. To simplify the discussion, following $[12,13]$ we assume that the local database $D$ remains unchanged during a run of $\tau$ until the output actions are committed. We also assume an infinite domain $\mathbf{D}$ of data values, on which the local databases, input messages and output actions are defined.

To specify an input sequence $\mathcal{I}$ we assume that the input schema $R_{i n}$ has a timestamp attribute ts of natural numbers, such that $I_{j}=\{t \mid t \in \mathcal{I} \wedge t[\mathrm{ts}]=j\}$ denotes the $j$-th input message of $\mathcal{I}$. In other words, $\mathcal{I}$ encodes an input sequence $I_{1}, \ldots, I_{n}$. We also assume that $R_{\text {in }}$ and $R_{\text {out }}$ simply consist of a single relation schema to simplify the presentation.

The SWS $\tau$ is defined with a set $Q$ of states to control its behavior. Associated with each state $q \in Q$ are an internal message register $\operatorname{Msg}(q)$ and an action register $\operatorname{Act}(q)$. We assume w.l.o.g. that $\operatorname{Msg}(q)$ and $\operatorname{Act}(q)$ store relations of schemas $R_{\text {in }}$ and $R_{\text {out }}$, respectively.

The queries in the transition and synthesis rules of $\tau$ are expressed in languages $\mathcal{L}_{\text {Msg }}$ and $\mathcal{L}_{\text {Act }}$, respectively.

SWS's. Putting these together, we define SWS's as follows.

Definition 2.1: Over schemas $\mathcal{R}, R_{\text {in }}, R_{\text {out }}$, a synthesized Web service $\tau$ in the class $\operatorname{SWS}\left(\mathcal{L}_{\mathrm{Msg}}, \mathcal{L}_{\text {Act }}\right)$ is defined to be $\left(Q, \delta, \sigma, q_{0}\right)$, where $Q$ is a finite set of states, $q_{0}$ is the start state, and $\delta, \sigma$ are sets of transition rules and synthesis rules, 
respectively, such that for each $q \in Q$, there exist a unique transition rule $\delta(q)$ and a unique synthesis rule $\sigma(q)$ :

$$
\begin{array}{ll}
\delta(q): & q \rightarrow\left(q_{1}, \phi_{1}\left(\bar{x}_{1}\right)\right), \ldots,\left(q_{k}, \phi_{k}\left(\bar{x}_{k}\right)\right) . \\
\sigma(q): & \operatorname{Act}(q) \leftarrow \psi(\bar{y}) .
\end{array}
$$

Here $q_{i} \in Q, \phi_{i}$ is a query in $\mathcal{L}_{\mathrm{Msg}}$ from $\mathcal{R}, R_{\text {in }}, \operatorname{Msg}(q)$ to $\operatorname{Msg}\left(q_{i}\right) ; \psi \in \mathcal{L}_{\text {Act }}$ is from $\operatorname{Act}\left(q_{1}\right), \ldots, \operatorname{Act}\left(q_{k}\right)$ to $\operatorname{Act}(q)$ if $k>0$, i.e., if the RHS of $\delta(q)$ is nonempty, and it is from $\mathcal{R}, R_{\text {in }}, \operatorname{Msg}(q)$ to $\operatorname{Act}(q)$ if $k=0$. Note that for $k>0$ we allow $\psi$ to only access previously computed action registers, while at final states $(k=0), \psi$ has access to external information stored in the local database, the current input, and its internal message register. As will be seen in Section 3, SWS's of this form are already expressive enough to express services supported by previous models. We assume that $q_{0}$ does not appear in the RHS of any rule. We refer to $q$ as the parent state of $q_{i}$, and $q_{i}$ as a successor state of $q$.

Note that $\delta(q)$ is parametrized with instances of $\mathcal{R}, R_{\text {in }}$ and $\operatorname{Msg}(q)$, i.e., $\delta$ is a finite representation of infinitely many transition rules; similarly for synthesis rules $\sigma$. We remark that the synthesis rules allow to model alternation in Web services, for which the need is advocated in Section 1.

Example 2.1: We define an SWS $\tau_{1}=\left(Q, \delta, \sigma, q_{0}\right)$ to specify the service described in Example 1.1. Assume that $R_{\text {in }}$ consists of attributes specifying user requirements, and an additional attribute tag with values of $a, h, t, c$, indicating whether an $R_{\text {in }}$ tuple is about airfare, hotel, ticket or car rental. Also assume that an $R_{\text {out }}$ tuple is of the form $R_{\text {out }}\left(\bar{x}_{a}, \bar{x}_{h}, \bar{x}_{t}, \bar{x}_{c}\right)$, with attribute lists $\bar{x}_{a}, \bar{x}_{h}, \bar{x}_{t}, \bar{x}_{c}$ for airfare, hotel, tickets and cars, respectively. Moreover, assume that $\mathcal{R}$ consists of relations $R_{a}, R_{h}, R_{t}, R_{c}$ containing information about airfares, hotels, tickets or cars, respectively. The set $Q$ of states includes $q_{0}, q_{a}, q_{h}, q_{c}, q_{t}$, and their transition and synthesis rules $\delta$ and $\sigma$ are given as follows:

$$
\begin{aligned}
& q_{0} \rightarrow\left(q_{a}, \phi_{a}\right),\left(q_{h}, \phi_{h}\right),\left(q_{t}, \phi_{t}\right),\left(q_{c}, \phi_{c}\right) \text {. } \\
& \operatorname{Act}\left(q_{0}\right) \leftarrow \psi_{0}(\bar{y}) \text {, where } \\
& \phi_{a}(\operatorname{tag}, \bar{x})=R_{i n}(\operatorname{tag}, \bar{x}) \wedge \operatorname{tag}=a / * \text { similarly for } \phi_{h}, \phi_{t}, \phi_{c} * / \\
& \psi_{0}\left(\bar{x}_{a}, \bar{x}_{h}, \bar{x}_{t}, \bar{x}_{c}\right)=\operatorname{Act}\left(q_{a}\right)\left(\bar{x}_{a},,_{-},,_{-}\right) \quad{ }^{*}{ }_{-} \text {for don't-care } * / \\
& \wedge \operatorname{Act}\left(q_{h}\right)\left({ }_{-}, \bar{x}_{h},,_{-},{ }_{-}\right) \wedge\left(\operatorname{Act}\left(q_{t}\right)\left({ }_{-},{ }_{-}, \bar{x}_{t}, \bar{x}_{c}\right)\right. \\
& \left.\vee \neg \exists \bar{x} \operatorname{Act}\left(q_{t}\right)(\bar{x}) \wedge \operatorname{Act}\left(q_{c}\right)\left({ }_{-},{ }_{-}, \bar{x}_{t}, \bar{x}_{c}\right)\right) \\
& q_{a} \rightarrow . \quad \operatorname{Act}\left(q_{a}\right) \leftarrow \psi_{a}(\bar{y}) . \quad /^{*} \text { similarly for } q_{h}, q_{t}, q_{c} * /
\end{aligned}
$$

Here $\psi_{a}$ (not shown) is an FO query that accesses $\operatorname{Msg}\left(q_{a}\right)$ and $R_{a}$, and returns airfares that satisfy the user's requirement. The register $\operatorname{Msg}\left(q_{a}\right)$ is computed by $\phi_{a}$ that simply selects input tuples for airfare; similarly for $\phi_{h}, \phi_{t}$ and $\phi_{c}$. Synthesis queries $\psi_{h}, \psi_{c}$ and $\psi_{t}$ (not shown) instantiate $\operatorname{Act}\left(q_{h}\right), \operatorname{Act}\left(q_{c}\right)$ and $\operatorname{Act}\left(q_{t}\right)$ with hotel room $\left(\bar{x}_{h}\right)$, car rental $\left(\bar{x}_{c}\right)$ and ticket sale $\left(\bar{x}_{t}\right)$ information, respectively. The output $\operatorname{Act}\left(q_{0}\right)$ is deterministically computed by $\psi_{0}$, which synthesizes actions from successor states of $q_{0}$, in favor of sale tickets, i.e., it copies $\operatorname{Act}\left(q_{t}\right)$ if it is nonempty, and takes $\operatorname{Act}\left(q_{c}\right)$ otherwise. It is nonempty only if airfares, hotels and either sale tickets or rental cars are found, as required.

One can readily extend $\tau_{1}$ and define $\tau_{2}$ to accommodate repeated user inquiries $\mathcal{I}$ for, e.g., airfare, as follows:

$$
\begin{gathered}
q_{a} \rightarrow\left(q_{a}, \phi_{a}\right),\left(q_{f}, \phi_{a}\right), \quad \operatorname{Act}\left(q_{a}\right) \leftarrow \psi_{a}^{\prime}(\bar{y}), \text { where } \\
\psi_{a}^{\prime}\left(\bar{x}_{a}, \bar{x}_{h}, \bar{x}_{t}, \bar{x}_{c}\right)=\operatorname{Act}\left(q_{a}\right)\left(\bar{x}_{a}, \bar{x}_{h}, \bar{x}_{t}, \bar{x}_{c}\right) \\
\vee \neg \exists \bar{x} \operatorname{Act}\left(q_{a}\right)(\bar{x}) \wedge \operatorname{Act}\left(q_{f}\right)\left(\bar{x}_{a}, \bar{x}_{h}, \bar{x}_{t}, \bar{x}_{c}\right) \\
q_{f} \rightarrow . \quad \operatorname{Act}\left(q_{f}\right) \leftarrow \psi_{f}(\bar{y}),
\end{gathered}
$$

where $\phi_{a}$ selects user requirements for airfare from input message $I_{j}$ in $\mathcal{I}$ as above, and $\psi_{f}$ finds airfares based on user inquiry $I_{j}$ by querying $\operatorname{Msg}\left(q_{a}\right)$ and $R_{a}$, similar to $\psi_{a}$. The synthesis query $\psi_{a}^{\prime}$ is in favor of actions $\operatorname{Act}\left(q_{a}\right)$ that are in turn synthesized at the "recursive" state $q_{a}$; in other words, it returns airfares that meet the latest user requirement $I_{j}$ in $\mathcal{I}$, if there is any, discarding earlier inquiry results.

Runs of SWS's. Following [5, 6], we specify the run of an SWS $\tau$ on a local database $D$ and an input message sequence $\mathcal{I}$ using a notion of execution trees. Each node $v$ in an execution tree is labeled with a state $q \in Q$, a timestamp $j$, a message register $\operatorname{Msg}(v)$ and an action register $\operatorname{Act}(v)$. Every step of the run rewrites an execution tree to another, following a step relation $\Rightarrow_{(\tau, D, \mathcal{I})}$.

The run starts from the root node $r$ of an execution tree $\xi$ labeled with $q_{0}$ and $j=0$, carrying $\operatorname{Msg}(r)=\emptyset$ whereas $\operatorname{Act}(r)=\perp$ (undefined). For two execution trees $\xi$ and $\xi^{\prime}$, $\xi \Rightarrow_{(\tau, D, \mathcal{I})} \xi^{\prime}$ if one of the following conditions holds.

Generating. If there is a leaf node $v$ of $\xi$ labeled $q, j, \operatorname{Msg}(v)$ $\overline{\text { and } \operatorname{Act}(v)}=\perp, \xi^{\prime}$ is obtained from $\xi$ as follows. Assume that $\mathcal{I}=I_{1}, \ldots, I_{n}$, and that the rules $\delta(q), \sigma(q)$ are

$$
q \rightarrow\left(q_{1}, \phi_{1}\left(\bar{x}_{1}\right)\right), \ldots,\left(q_{k}, \phi_{k}\left(\bar{x}_{k}\right)\right) . \operatorname{Act}(q) \leftarrow \psi(\bar{y}) .
$$

(1) If either $j>n$ or $\operatorname{Msg}(v)$ is empty, then $\xi^{\prime}$ is obtained from $\xi$ by setting $\operatorname{Act}(v)=\emptyset$. A special case is when $q=$ $q_{0}$, at the root $r$ : even if $\operatorname{Msg}(r)$ is empty, we allow the construction to proceed as follows if $\mathcal{I}$ is nonempty.

(2) Otherwise, if $k>0$, then $\xi^{\prime}$ is obtained from $\xi$ by spawning $k$ children $u_{1}, \ldots, u_{k}$ for $v$, in parallel. More specifically, for each $i \in[1, k]$, a distinct node $u_{i}$ is created as the $i$-th child of $v$, labeled $q_{i}$ and $j+1$ such that $\operatorname{Msg}\left(u_{i}\right)$ carries $\phi_{i}\left(D, I_{j}, \operatorname{Msg}(v)\right)$ but leaving $\operatorname{Act}\left(u_{i}\right)=\perp$. Here $\phi_{i}\left(D, I_{j}, \operatorname{Msg}(v)\right)=\left\{\bar{d} \mid\left(D, I_{j}, \operatorname{Msg}(v)\right) \models \phi_{i}(\bar{d})\right\}$.

Gathering. Action register $\operatorname{Act}(v)$ is populated as follows.

(3) If $k=0$, i.e., the RHS of $\delta(q)$ is empty and $v$ is a leaf node, then $\xi^{\prime}$ is obtained from $\xi$ by letting $\operatorname{Act}(v)=$ $\psi\left(D, I_{j}, \operatorname{Msg}(v)\right)$, where $\psi(\bar{y})$ is the query in the synthesis rule $\sigma(q)$, producing actions by accessing $\operatorname{Msg}(v), I_{j}$ and $D$.

(4) Otherwise, if for all the children $u_{1}, \ldots, u_{k}$ of $v$, $\operatorname{Act}\left(u_{i}\right) \neq \perp$, then $\xi^{\prime}$ is obtained from $\xi$ by setting $\operatorname{Act}(v)=\psi\left(\operatorname{Act}\left(u_{1}\right), \ldots, \operatorname{Act}\left(u_{k}\right)\right)$, i.e., the query $\psi$ synthesizes actions from $\operatorname{Act}\left(u_{i}\right)$. In other words, the synthesis is halted at $v$ until $\operatorname{Act}\left(u_{i}\right)$ is available for all $i \in[1, k]$.

Observe that an execution tree stops spawning new nodes at $v$ if (a) the input message $\mathcal{I}$ is entirely consumed (i.e., $j>n)$, (b) it receives an empty internal message $\operatorname{Msg}(v)$ (if $q \neq q_{0}$ ), or (c) $v$ is at a "final state" indicated by the transition rule (with an empty RHS). While the tree is generated top-down, action gathering and synthesis are conducted bottom-up. The run takes one sweep: each node is accessed at most twice, for node generation and action gathering, respectively.

Output. Denote by $\Rightarrow_{(\tau, D, \mathcal{I})}^{*}$ the reflexive and transitive closure of $\Rightarrow_{(\tau, D, \mathcal{I})}$. The result of the run of the SWS $\tau$ on $(D, \mathcal{I})$ is an execution tree $\xi$ such that $r \Rightarrow^{*} \xi$, where $r$ is the root labeled $q_{0}$ and timestamp 1, and there exists no node $v$ in $\xi$ with $\operatorname{Act}(v)=\perp$. The output of the $\tau$-run, denoted by $\tau(D, \mathcal{I})$, is the content of the action register $\operatorname{Act}(r)$.

Example 2.2: Recall the SWS $\tau_{1}$ defined in Example 2.1. Given an instance $D=\left(I_{a}, I_{h}, I_{c}, I_{t}\right)$ of the local database 
schema $\mathcal{R}$ and a sequence of input messages $\mathcal{I}=I_{1}, \ldots, I_{n}$, the result $\tau_{1}(D, \mathcal{I})$ of the run of $\tau_{1}$ is a relation that is nonempty iff the conditions 1-3 given in Example 1.1 are all satisfied. The execution tree of the run is constructed as follows, starting from the root node $r$. Four children $v_{a}, v_{h}, v_{t}, v_{c}$ of $r$ are created in parallel. The node $v_{a}$ is a leaf of the tree labeled with ts $=1$, state $q_{a}$ and two registers. Its message register $\operatorname{Msg}\left(v_{a}\right)$ is set by $\phi_{a}\left(I_{1}\right)$ with input tuples for airfare, followed by the instantiation of the action register $\operatorname{Act}\left(v_{a}\right)$ by $\psi_{a}\left(I_{a}, \operatorname{Msg}\left(v_{a}\right)\right)$ with tuples for booking flights that meet the user request, if any; similarly for $v_{h}, v_{t}$ and $v_{c}$. As soon as values are assigned to $\operatorname{Act}\left(v_{a}\right), \operatorname{Act}\left(v_{h}\right), \operatorname{Act}\left(v_{t}\right)$ and $\operatorname{Act}\left(v_{c}\right)$, the synthesis query $\psi_{0}\left(\operatorname{Act}\left(v_{a}\right), \operatorname{Act}\left(v_{h}\right), \operatorname{Act}\left(v_{c}\right), \operatorname{Act}\left(v_{t}\right)\right)$ populates $\operatorname{Act}(r)$, as described in Example 2.1. The content of register Act $(r)$ is the output. Here $I_{2}, \ldots, I_{n}$ are not consumed by $\tau_{1}$; i.e., it suffices for $\tau_{1}$ to produce output when $\mathcal{I}$ consists of a single input message.

Note that the synthesis query $\psi_{0}$ makes a deterministic decision between $\operatorname{Act}\left(v_{c}\right)$ and $\operatorname{Act}\left(v_{t}\right)$, and it specifies a conjunctive condition on reservations of flight, hotel and local arrangement (either car rental or tickets). To simplify the discussion, while user requests for flight, hotel, tickets and cars are usually given in different input relations, we encode them in a single relation.

The SWS $\tau_{2}$ behaves similarly, except that it can accept an input sequence $\mathcal{I}=I_{1}, \ldots, I_{n}$ of an unbounded length $n$. Here below $v_{a}$ is a chain of node pairs $\left(v_{j}, f_{j}\right)$, which indicate the processing of user inquiry $I_{j}$ for airfare, for $j \in[2, n]$. The synthesis query $\psi_{a}$ assures that the nonempty $\operatorname{Act}\left(f_{j}\right)$ with the largest $j$ is used to populate $\operatorname{Act}\left(v_{a}\right)$.

SWS classes. To characterize prior models developed for specifying Web services, we study various $\operatorname{SWS}\left(\mathcal{L}_{\text {Msg }}, \mathcal{L}_{\text {Act }}\right)$ classes, where $\mathcal{L}_{\text {Msg }}$ and $\mathcal{L}_{\text {Act }}$ are the languages for expressing queries embedded in transition and synthesis rules, respectively. As suggested by condition (3) in the definition of execution trees, we assume $\mathcal{L}_{\text {Act }}$ to be at least as expressive as $\mathcal{L}_{\text {Msg }}$, but also consider special cases when $\mathcal{L}_{\text {Act }}$ is not as powerful as $\mathcal{L}_{\mathrm{Msg}}$. In particular, we study the following classes: SWS(FO, FO), SWS(CQ, UCQ) and SWS(PL, PL), for first-order logic (FO), conjunctive queries (CQ), union of conjunctive queries (UCQ), and propositional logic (PL), with ' $=$ ' and inequality ' $\neq$ ' in CQ and UCQ. For example, $\tau_{1}, \tau_{2}$ given in Example 2.1 are in $\operatorname{SWS}(\mathrm{FO}, \mathrm{FO})$. We do not consider SWS(CQ, CQ) since not many interesting services can be specified in the absence of union in synthesis rules.

As will be seen shortly, Web services of the Roman model [6] can be modeled as a special case of SWS(PL, PL). Unlike CQ, UCQ or FO, a PL-formula is defined over propositional variables. An SWS $\tau$ in SWS(PL, PL) aims to specify a service that is not data-driven, i.e., on empty local databases. An input message $I_{j}$ of $R_{i n}$ for $\tau$ is a truth assignment, represented as a set of propositional variables such that a variable $X$ is true iff $X \in I_{j}$. Note that a sequence $\mathcal{I}$ of $R_{\text {in }}$ instances can encode a string over an alphabet found in FSA abstractions of Web services. Message and action registers, as well as output $\mathcal{O}$ of $\tau$ consist of a single truth value true or false, along the same lines as FSA abstractions.

For each $\operatorname{SWS}\left(\mathcal{L}_{\mathrm{Msg}}, \mathcal{L}_{\mathrm{Act}}\right)$, we also study its subclass $\mathrm{SWS}_{\mathrm{nr}}\left(\mathcal{L}_{\mathrm{Msg}}, \mathcal{L}_{\mathrm{Act}}\right)$ consisting of all nonrecursive SWS's, defined in terms of a notion of dependency graphs as follows. The dependency graph $G_{\tau}$ of an SWS $\tau$ is a graph in which each $q \in Q$ is a distinct node, and there is an edge from $q$ to $q_{i}$ iff $q_{i}$ is in the RHS of the transition rule of $q$. An SWS $\tau$ is said to be recursive if the graph $G_{\tau}$ is cyclic. For instance, $\tau_{2}$ of Example 2.1 is recursive, but $\tau_{1}$ is nonrecursive. Nonrecursive SWS's aim to model services that respond to a user input within a bounded number of computation steps. As will be seen in Section 5, a nonrecursive SWS may be repeatedly invoked by a composite service (mediator) for an unbounded number of times to process various inputs.

\section{Specifying Web Services as SWS's}

In this section we show that the behaviors of Web services defined in terms of FSA or transducer abstractions can be specified by various SWS classes. We consider two representative models: the Roman model [6] and the peer model of [13]. The Roman model is an FSA abstraction for specifying non-data-driven services, and peers of [13] are a transducer abstraction for specifying data-driven Web services.

For each of these models, we identify an SWS class $\mathcal{S}$ and give two functions $f_{\tau}$ and $f_{I}$, such that (a) for each service $\omega$ defined in the model, $f_{\tau}(\omega)$ is an SWS $\tau$ in $\mathcal{S}$, and (b) for any input sequence $\mathcal{I}$ of $\omega, f_{I}(\mathcal{I})$ is an input sequence $\mathcal{I}$ for $\tau$ such that for any local database $D, \tau(D, \mathcal{I})$ yields the same output as $\omega$ on $\mathcal{I}$ and $D$.

The Roman model [6]. A service in the model is expressed as a DFA (or an NFA for a composite service), in which the alphabet is interpreted as a set of actions. It takes a string over the alphabet and returns true if the string leads to a final state, indicating that the service legally terminates.

One can define a function $f_{\tau}$ that, given such a DFA $\omega$, derives $\tau$ in SWS(PL, PL). The SWS $\tau$ includes all the states of $\omega$ and an additional $q_{f}$. The transition rule for each state $q$ from $\omega$ collects all transition rules for $q$ in $\omega: q \rightarrow$ $\left(q_{1}, \phi_{1}\right), \ldots,\left(q_{k}, \phi_{k}\right),\left[\left(q_{f}, \phi_{f}\right)\right]$, where $q \rightarrow q_{i}$ is a transition in $\omega$ upon receiving a letter $a_{i}$, and $\phi_{i}$ simply checks whether the input message is $a_{i}$; moreover, if $q$ is a final state, it has $q_{f}$ as a successor state, for which $\phi_{f}$ checks whether the input is a special delimiter \#. The synthesis rule for $q_{f}$ is $\operatorname{Act}\left(q_{f}\right) \leftarrow \operatorname{Msg}\left(q_{f}\right)$, while the synthesis query for $q$ is the disjunction of $\operatorname{Act}\left(q_{i}\right)$ of all $q_{i}$. The function $f_{I}$ is defined such that given a string $\mathcal{I}$ over the alphabet, it derives $\mathcal{I}$ that augments each letter with its index in $\mathcal{I}$ and adds the delimiter \# to the end of $\mathcal{I}$. One can easily see that for any $\mathcal{I}, \omega(\mathcal{I})=\tau(D, \mathcal{I})$, where $D$ is an empty local database. Note that $\tau$ only needs disjunction in its synthesis rules.

One can also define $\tau$ in $\mathrm{SWS}(\mathrm{CQ}, \mathrm{UCQ})$ that returns the empty set if $\mathcal{I}$ is not accepted by $\omega$, and the same $\mathcal{I}$ otherwise. That is, $\tau$ defers the commitment of the actions until it confirms that all actions in $\mathcal{I}$ can be legally conducted.

The peer model [13]. A peer of [13] is characterized by (1) a local database $D$ that is fixed during execution; (2) a set $\mathcal{S}$ of state relations keeping track of updates to $D ;(3)$ a set $\mathcal{I}$ of user input relations; (4) a set $\mathcal{A}$ of action relations; (5) a set of in-queues $\mathcal{Q}_{i}$ and out-queues $\mathcal{Q}_{o}$, in which elements are input and output message relations from and to other services, respectively; and (6) a set of rules defined as FO queries on $D, \mathcal{S}, \mathcal{Q}_{i}$, current and previous $\mathcal{I}$. At each step the peer uses the rules to produce actions, updates and output messages to be included in $\mathcal{A}, \mathcal{S}$ and $\mathcal{Q}_{o}$, respectively.

A function $f_{\tau}$ can be defined that derives from a peer $\omega$ an SWS $\tau$ in SWS(FO, FO). The SWS $\tau$ encodes $\mathcal{I}$ and 


\begin{tabular}{|l||c|c|c|}
\hline & Non-emptiness & Validation & Equivalence \\
\hline \hline SWS $_{\mathrm{nr}}(\mathrm{FO}, \mathrm{FO})$ & undecidable & undecidable & undecidable \\
\hline SWS(CQ, UCQ $)$ & EXPTIME-complete & undecidable & undecidable \\
\hline SWS $\mathrm{nr}(\mathrm{CQ}, \mathrm{UCQ})$ & PSPACE-complete & NEXPTIME-complete & conEXPTIME-complete \\
\hline SWS(PL, PL $)$ & PSPACE-complete & PSPACE-complete & PSPACE-complete \\
\hline SWS $\mathrm{nr}(\mathrm{PL}, \mathrm{PL})$ & NP-complete & NP-complete & conP-complete \\
\hline
\end{tabular}

Table 1: Complexity of decision problems

$\mathcal{Q}_{i}$ in terms of a single input schema $R_{i n}$, and $\mathcal{S}, \mathcal{A}$ and $\mathcal{Q}_{o}$ with a single $R_{\text {out }}$. It is recursively defined, with three states $q_{0}, q_{s}, q_{f}$. The transition rule for $q_{f}$ has an empty RHS, while for $q_{0}, q_{s}$, they are $q_{0} \rightarrow\left(q_{s}, \phi\right),\left(q_{f}, \phi\right)$ and $q_{s} \rightarrow$ $\left(q_{s}, \phi\right),\left(q_{f}, \phi\right)$, where $\phi$ is an FO query that combines all queries in the rules of $\omega$. The synthesis rule for $q_{f}$ copies the query result to the output; the synthesis rules for $q_{0}$ and $q_{s}$ take unions of actions generated by their successor states.

The peer $\omega$ produces an output at each step of execution. To cope with this interactive behavior, we define function $f_{I}$ such that for each input sequence $\bar{I}=I_{1}, \ldots, I_{n}$ for $\omega$, it returns $\mathcal{I}=I_{1}, \#, I_{1}, I_{2}, \#, \ldots, \#, I_{1}, \ldots, I_{n}, \#$. The SWS $\tau$ produces an output whenever a delimiter \# is encountered. Then for any $\bar{I}$ and any database $D, \tau(D, \mathcal{I})$ yields the same output as $\omega(\bar{I}, D)$ at each step $j \in[1, n]$.

Other models. As observed in [13], services supported by the Colombo model [5] or expressed as guarded automata of [15] can also be expressed as peers of [13]. As a result, one can also use SWS(FO, FO) to study the behaviors of the Colombo services and guarded automata [15].

\section{Complexity of Synthesized Web Services}

In this section we investigate static analyses of SWS's. For a class $\operatorname{SWS}\left(\mathcal{L}_{\mathrm{Msg}}, \mathcal{L}_{\text {Act }}\right)$, we study the following decision problems. As remarked in Section 1, these problems are of both theoretical and practical interest.

- The non-emptiness problem is to determine, given an SWS $\tau$ in the class defined over a database schema $\mathcal{R}$ and an input schema $R_{\text {in }}$, whether there exist an instance $D$ of $\mathcal{R}$ and a sequence $\mathcal{I}$ of $R_{\text {in }}$ instances such that $\tau(D, \mathcal{I})$ is nonempty. That is, whether or not $\tau$ can generate actions at all.

- The validation problem is to determine, given $\tau$ in the class over $\mathcal{R}$ and $R_{\text {in }}$, and an instance $\mathcal{O}$ of its external schema $R_{\text {out }}$, whether or not there exist an instance $D$ of $\mathcal{R}$ and a sequence $\mathcal{I}$ of $R_{\text {in }}$ instances such that $\tau(D, \mathcal{I})=\mathcal{O}$. That is, whether $\tau$ is capable of producing the desired actions.

- The equivalence problem is to determine, given two SWS's $\tau_{1}$ and $\tau_{2}$ in this class, both defined over the same $\mathcal{R}$, $R_{\text {in }}$ and $R_{\text {out }}$, whether or not $\tau_{1}(D, \mathcal{I})=\tau_{2}(D, \mathcal{I})$ for all instances $D$ of $\mathcal{R}$ and sequences $\mathcal{I}$ of $R_{\text {in }}$ instances. With a cost model, the equivalence analysis helps users decide what service to choose from a set of available services.

We establish matching complexity bounds on these problems for the SWS classes given in Section 2, recursive or not. The results are summarized in Table 1 . They tell us the following. First, for nonrecursive $\mathrm{SWS}_{\mathrm{nr}}(\mathrm{FO}, \mathrm{FO})$ all these problems are already beyond reach. Second, while compiletime equivalence and validation analyses are still infeasible for SWS(CQ, UCQ), they are decidable for $\mathrm{SWS}_{\mathrm{nr}}(\mathrm{CQ}$, UCQ) in conEXPTIME and NEXPTIME, respectively. That is, the absence of recursion makes our lives easier, although still not easy enough. Third, the equivalence problem for SWS(PL, PL) has the same complexity as its NFA and AFA counterparts, and its emptiness analysis is no harder than that for AFA.

Theorem 4.1: The non-emptiness, validation and equivalence problems are

1. undecidable for $\mathrm{SWS}(\mathrm{FO}, \mathrm{FO})$ and $\mathrm{SWS}_{\mathrm{nr}}(\mathrm{FO}, \mathrm{FO})$;

2. EXPTIME-complete, undecidable and undecidable for $\mathrm{SWS}(\mathrm{CQ}, \mathrm{UCQ})$; but for $\mathrm{SWS}_{\mathrm{nr}}(\mathrm{CQ}, \mathrm{UCQ})$ they become PSPACE-complete, NEXPTIME-complete and cONEXPTIME-complete, respectively;

3. PSPACE-complete for SWS(PL,PL); for $\mathrm{SWS}_{\mathrm{nr}}(\mathrm{PL}, \mathrm{PL})$ these problems become NP-complete, NP-complete and conP-complete, respectively.

Proof. (1) For $\mathrm{SWS}_{\mathrm{nr}}(\mathrm{FO}, \mathrm{FO})$, the undecidability of these problems is proved by reduction from the satisfiability problem for FO queries, which is undecidable (cf. [1]).

(2) For SWS(CQ, UCQ), the validation and equivalence problems are shown undecidable by reduction from the non-emptiness problem for deterministic finite 2-head machines, which is undecidable [28]. The lower bound on nonemptiness analysis is by reduction from the problem for deciding whether a single ground fact, single rule datalog program (sirup) accepts a goal, an EXPTIME-complete problem [19]. Its upper bound is by reduction to the emptiness problem for a form of tree automata [8] that characterize the execution trees of SWS's; while the latter can be checked in PTIME, the reduction takes EXPTIME.

For $\mathrm{SWS}_{\mathrm{nr}}(\mathrm{CQ}, \mathrm{UCQ})$, we show the lower bound on nonemptiness analysis by reduction from Q3SAT, a PSPACEcomplete problem (cf. [17]), and the upper bound by giving a PSPACE checking algorithm. The lower bound on the equivalence problem is by reduction from the problem for checking whether a nondeterministic Turing machine (NTM) halts in exponential steps on a given input. Similarly we prove the lower bound on validation analysis. We develop a coNEXPTIME checking algorithm for $\mathrm{SWS}_{\mathrm{nr}}(\mathrm{CQ}, \mathrm{UCQ})$ equivalence, by extending the containment algorithm of [22] for nonrecursive datalog with inequality. We show that validation analysis is in NEXPTIME by establishing a small model property: given an SWS $\tau$ and output $\mathcal{O}$, if there exist a database $D$ and an input sequence $\mathcal{I}$ such that $\tau(D, \mathcal{I})=\mathcal{O}$, then there exist such $D$ and $\mathcal{I}$ bounded by an exponential in the size of $\mathcal{O}$ and $\tau$. Since evaluating $\tau$ on such a $(D, \mathcal{I})$ and checking whether $\tau(D, \mathcal{I})=\mathcal{O}$ are both in Exptime, a NEXPTIME algorithm is immediate.

(3) For SWS(PL, PL), the non-emptiness problem coincides with the validation problem, and is PTIME-equivalent to the complement of the equivalence problem. The lower bound on the non-emptiness problem follows from the PSPACE lower bound on the emptiness problem for AFA [32], which can be expressed in SWS(PL, PL), in PTIME. For the upper bound, one can check non-emptiness in PSPACE along the same lines as AFA non-emptiness checking.

For $\mathrm{SWS}_{\mathrm{nr}}(\mathrm{PL}, \mathrm{PL})$, the lower bound is proved by reduction from SAT, which is NP-complete (cf. [17]), and the upper 
bound by noting that the non-emptiness checking algorithm for SWS(PL, PL) is in NP on nonrecursive SWS's.

Special cases. For services defined as NFA (resp. DFA) in, e.g., the Roman model, the complexity on the equivalence and non-emptiness problems is known to be PSPACEcomplete (resp. NLOGSPACE-complete) and NLOGSPACEcomplete, respectively [32]. Note that the validation problem studied here is quite different from the membership problem for NFA (resp. DFA). We remark that for SWS(PL, PL) and for services defined as NFA (resp. DFA), the validation problem coincides with the non-emptiness problem. In contrast, it is entirely different from non-emptiness for data-driven services in SWS(FO, FO) and SWS(CQ, UCQ).

\section{Composition Synthesis}

We next focus on composition synthesis of SWS's. We first formalize the notion of SWS mediators, and then provide complexity bounds on composition synthesis for various classes of SWS's identified in Section 2. Our main conclusion is that the composition synthesis problem is nontrivial: it is undecidable or highly intractable for data-driven or recursive SWS's. In light of the undecidability results we identify decidable cases of data-driven or recursive SWS's.

\subsection{SWS Mediators and Composition}

A mediator coordinates available services by routing the output of one service to the input of another, in order to deliver a requested service by invoking available services as component services $[7,5]$. It receives and redirects messages, but does not directly access local databases. An SWS mediator also synthesizes actions produced by component services.

We parametrize SWS mediators with languages $\mathcal{L}_{\text {Act }}$ for expressing action synthesis. For a fixed $\mathcal{L}_{\text {Act }}$, we define a class of mediators, denoted by $\operatorname{MDT}\left(\mathcal{L}_{\text {Act }}\right)$, in the same way as SWS's except that component services are embedded in transition rules and are treated as "oracle queries".

Definition 5.1: Over a set $S$ of available SWS's defined on schemas $\mathcal{R}, R_{\text {in }}, R_{\text {out }}$, an SWS mediator in $\operatorname{MDT}\left(\mathcal{L}_{\text {Act }}\right)$ is defined to be $\pi=\left(Q, \delta, \sigma, q_{0}\right)$, where $Q$ and $q_{0}$ are as given in Definition 2.1, and $\delta$ is a set of transition rules such that for each $q \in Q$, there exists a unique rule of the form

$$
q \rightarrow\left(q_{1}, \operatorname{eval}\left(\tau_{1}\right)\right), \ldots,\left(q_{k}, \operatorname{eval}\left(\tau_{k}\right)\right) .
$$

Here $q_{i} \in Q, \tau_{i} \in S$, where $\tau_{i}$ is referred to as a component service of $\pi$, and eval $\left(\tau_{i}\right)$ is an evaluation operator whose semantics will be explained below. The synthesis rule $\sigma(q)$ is defined via a query $\psi \in \mathcal{L}_{\text {Act }}$ as in SWS's, except that if $k=0, \psi$ is from $\operatorname{Msg}(q)$ to $\operatorname{Act}(q)$ without accessing database and input.

To simplify the presentation we assume that SWS's in $S$ are defined over the same input, external and database schemas $\mathcal{R}, R_{\text {in }}$ and $R_{\text {out }}$. This does not lose generality for composition synthesis since one can take $\mathcal{R}$ as the collection of local database schemas of all component SWS's in $S$, and unify $R_{\text {in }}$ (resp. $R_{\text {out }}$ ) by e.g., taking outer union of input (resp. external) relations of SWS's in $S$.

Runs of mediators. Like SWS's, a mediator $\pi$ takes as input an instance $D$ of the database schema $\mathcal{R}$ and a sequence $\mathcal{I}$ of instances of the input schema $R_{i n}$; the run of $\pi$ on $(D, \mathcal{I})$ returns an instance of the external schema $R_{\text {out }}$. A step relation $\Rightarrow_{(\pi, D, \mathcal{I})}$ is defined for runs of $\pi$ as for SWS's.
There are, however, subtle differences in the run of $\pi$ on $D$ and $\mathcal{I}$. More specifically, for execution trees $\xi$ and $\xi^{\prime}$, cases (2) and (3) of the transition $\xi \Rightarrow_{(\pi, D, \mathcal{I})} \xi^{\prime}$ described in Section 2 are now modified as follows. Consider a leaf node $v$ in $\xi$, labeled with $q, j, \operatorname{Msg}(v)$ and $\operatorname{Act}(v)=\perp$. Assume that $\mathcal{I}=I_{1}, \ldots, I_{n}$, and that $\delta(q)$ and $\sigma(q)$ are

$$
q \rightarrow\left(q_{1}, \operatorname{eval}\left(\tau_{1}\right)\right), \ldots,\left(q_{k}, \operatorname{eval}\left(\tau_{k}\right)\right), \operatorname{Act}(q) \leftarrow \psi(\bar{y}) .
$$

(2) If $k>0$, then as before, $\xi^{\prime}$ is obtained from $\xi$ by spawning $k$ children $u_{1}, \ldots, u_{k}$ for $v$, in parallel, such that for each $i \in[1, k]$, a distinct node $u_{i}$ is created as the $i$-th child of $v$, labeled with $q_{i}$ and $\operatorname{Act}\left(u_{i}\right)=\perp$. In contrast to its SWS counterpart, here $\operatorname{Msg}\left(u_{i}\right)$ is instantiated with the result of $\operatorname{eval}\left(\tau_{i}\right)$. That is, eval $\left(\tau_{i}\right)$ is equal to $\tau_{i}\left(D, \mathcal{I}^{j}\right)$ of the component SWS $\tau_{i}$ on $D$ and $\mathcal{I}^{j}$, where $\mathcal{I}^{j}=I_{j}, \ldots, I_{n}$. That is, $\tau_{i}$ operates on $I_{j}, \ldots, I_{n}$, runs to completion, and its output is used as the internal message of $u_{i}$. Moreover, in the run of $\tau_{i}$, the message register of the start state of $\tau_{i}$ is instantiated with $\operatorname{Msg}(v)$. Furthermore, $u_{i}$ is labeled with timestamp $l_{i}+1$, where $l_{i}$ is the maximum timestamp in the result execution tree of the run of $\tau_{i}$ on $\left(D, \mathcal{I}^{j}\right)$. In other words, $I_{l_{i}+1}$ indicates the first input message that has not been "consumed" by the run of $\tau_{i}$.

(3) If $k=0$, then the synthesis query $\psi$ can access $\operatorname{Msg}(v)$ but neither the database $D$ nor the input message $I_{j}$, i.e., $\xi^{\prime}$ is obtained from $\xi$ by letting $\operatorname{Act}(v)=\psi(\operatorname{Msg}(v))$.

The rest of the run is defined as for SWS's, as described in Section 2. The commitment of actions produced by the component services is deferred to the end of the run of $\pi$. The output of the run of $\pi$ on $(D, \mathcal{I})$ is denoted by $\pi(D, \mathcal{I})$.

We can compare SWS's and mediators as follows. Consider a mediator $\pi$ over a set of component SWS's defined on schemas $\mathcal{R}, R_{\text {in }}, R_{\text {out }}$, and an SWS $\tau$ defined on the same $\mathcal{R}, R_{\text {in }}, R_{\text {out }}$. We say that $\pi$ and $\tau$ are equivalent, denoted by $\pi \equiv \tau$, if $\pi(D, \mathcal{I})=\tau(D, \mathcal{I})$ for any instance $D$ of $\mathcal{R}$ and any input sequence $\mathcal{I}$ of $R_{\text {in }}$ instances.

We study $\operatorname{MDT}\left(\mathcal{L}_{\text {Act }}\right)$ for $\mathcal{L}_{\text {Act }}$ ranging over PL, UCQ and FO. We denote by $\operatorname{MDT}_{\mathrm{nr}}\left(\mathcal{L}_{\text {Act }}\right)$ the class of nonrecursive mediators in $\operatorname{MDT}\left(\mathcal{L}_{\mathrm{Act}}\right)$ defined in the same way as their nonrecursive SWS counterparts. Note that component SWS's embedded in a nonrecursive mediator may be recursive.

Example 5.1: Recall SWS $\tau_{1}$ and $R_{\text {out }}\left(\bar{x}_{a}, \bar{x}_{h}, \bar{x}_{t}, \bar{x}_{c}\right)$ from Example 2.1. Suppose that we are given a set $S$ of component services consisting of $\tau_{a}$ for flight reservations, $\tau_{h c}$ for reserving both hotel rooms and rental cars, and $\tau_{h t}$ for reserving both hotel and Disney tickets. One can define a mediator $\pi_{1}=\left(Q_{1}, \delta_{1}, \sigma_{1}, q_{1}\right)$, where $Q_{1}=\left\{q_{1}, q_{a}, q_{h c}, q_{h t}\right\}$, and transition and synthesis rules $\delta_{1}$ and $\sigma_{1}$ are as follows.

$$
\begin{aligned}
& q_{1} \rightarrow\left(q_{a}, \operatorname{eval}\left(\tau_{a}\right)\right),\left(q_{h c}, \operatorname{eval}\left(\tau_{h c}\right)\right),\left(q_{h t}, \text { eval }\left(\tau_{h t}\right)\right), \\
& \operatorname{Act}\left(q_{1}\right) \leftarrow \psi_{1}(\bar{y}), \text { where } \\
& \psi_{1}\left(\bar{x}_{a}, \bar{x}_{h}, \bar{x}_{t}, \bar{x}_{c}\right)=\operatorname{Act}\left(q_{a}\right)\left(\bar{x}_{a},,_{-},,_{-}\right) \wedge \\
& \quad\left(\operatorname{Act}\left(q_{h t}\right)\left(-, \bar{x}_{h}, \bar{x}_{t}, \bar{x}_{c}\right) \vee\right. \\
& \left.\quad \neg \exists \bar{y} \operatorname{Act}\left(q_{h t}\right)(\bar{y}) \wedge \operatorname{Act}\left(q_{h c}\right)\left({ }_{-}, \bar{x}_{h}, \bar{x}_{t}, \bar{x}_{c}\right)\right) \\
& q_{a} \rightarrow . \quad \operatorname{Act}\left(q_{a}\right) \leftarrow \psi_{a}(\bar{y}) /{ }^{*} \text { similarly for } q_{h c} \text { and } q_{h t} * / \\
& \text { where } \psi_{a}\left(\bar{x}_{a},,_{-},-\right)=\operatorname{Msg}\left(q_{a}\right)\left(\bar{x}_{a},,_{-},,_{-}\right)
\end{aligned}
$$

Here $\tau_{a}$ is invoked by $q_{1}$ to find airfares, and its output is used to instantiate $\operatorname{Act}\left(q_{a}\right)$; similarly for $\tau_{h c}$ and $\tau_{h t}$. The outputs of these component services are synthesized by $\psi_{1}$, which is in favor of Disney tickets. One can verify that $\tau_{1}$ and $\pi_{1}$ are equivalent provided that (a) $\tau_{a}$ finds all flights that $\phi_{a}$ of Example 2.1 can find, (b) both $\tau_{h c}$ and $\tau_{h t}$ find all hotel rooms that $\phi_{a}$ finds, and (c) $\tau_{h t}\left(\right.$ resp. $\tau_{h c}$ ) finds all 
tickets (resp. cars) that $\phi_{t}$ (resp. $\phi_{c}$ ) finds, and vice versa. This mediator is in the class $\operatorname{MDT}_{\mathrm{nr}}(\mathrm{FO})$.

Composition synthesis. In the reminder of the section we study the following family of composition synthesis problems, referred to $\operatorname{CP}(\mathcal{G}, \mathcal{M}, \mathcal{C})$, where $\mathcal{G}, \mathcal{M}$ and $\mathcal{C}$ are called the goal (user-requested) service, mediator and component (available) service classes, respectively. Here $\mathcal{G}$ and $\mathcal{C}$ are either $\operatorname{SWS}\left(\mathcal{L}_{\mathrm{Msg}}, \mathcal{L}_{\text {Act }}\right)$ or $\operatorname{SWS}_{\mathrm{nr}}\left(\mathcal{L}_{\mathrm{Msg}}, \mathcal{L}_{\text {Act }}\right)$, and $\mathcal{M}$ is either $\operatorname{MDT}\left(\mathcal{L}_{\text {Act }}\right)$ or $\operatorname{MDT}_{\text {nr }}\left(\mathcal{L}_{\text {Act }}\right)$. We consider $\left(\mathcal{L}_{\text {Msg }}, \mathcal{L}_{\text {Act }}\right)$ ranging over (PL, PL), (CQ, UCQ) and (FO, FO).

Given a goal SWS $\tau \in \mathcal{G}$ and a finite set $S \subset \mathcal{C}$ of component services, all defined over the same schemas $\mathcal{R}, R_{\text {in }}$ and $R_{\text {out }}, \operatorname{CP}(\mathcal{G}, \mathcal{M}, \mathcal{C})$ is to determine whether or not there exists a mediator $\pi \in \mathcal{M}$ over $S$ such that $\pi \equiv \tau$.

\subsection{Complexity of Composition Synthesis}

There is a connection between SWS composition and query rewriting using views. For a fixed relational query language $\mathcal{L}$, the problem of equivalent query rewriting using views (cf. [20]) is to determine, given a query $\psi \in \mathcal{L}$ and a set of view definitions $\mathcal{V}$ in $\mathcal{L}$, whether or not there exists an equivalent rewriting of $\psi$, i.e., a query $\psi^{\prime} \in \mathcal{L}$ such that $\psi$ and $\psi^{\prime}$ are equivalent, and $\psi^{\prime}$ accesses only relations in $\mathcal{V}$. A query $\psi^{\prime}$ is referred to as a maximally-contained rewriting of $\psi$ if $\psi^{\prime}$ is contained in $\psi, \psi^{\prime}$ accesses only relations in $\mathcal{V}$, and there exists no $\psi_{1} \in \mathcal{L}$ such that $\psi^{\prime}$ is contained in $\psi_{1}, \psi_{1}$ is contained in $\psi$, and $\psi_{1}$ accesses only relations in $\mathcal{V}$. For $\mathcal{L}$ ranging over datalog, UCQ, CQ and regular path queries, the problems of finding equivalent and maximallycontained rewritings have been studied (e.g., [8, 3, 14, 23]). The connection between these problems and SWS composition is evident if we treat the goal service $\tau \in \mathcal{G}$ and mediator $\pi \in \mathcal{M}$ as queries, and component services $S \subset \mathcal{C}$ as views.

We study SWS composition synthesis by capitalizing on this connection. It is, however, nontrivial to apply prior results on query rewriting to SWS composition. First, SWS's may not be expressible in any well-studied query languages. Second, for SWS's that can be expressed in a query language, the rewriting problem for that language may not be settled. For example, although SWS's in $\mathrm{SWS}_{\mathrm{nr}}(\mathrm{CQ}, \mathrm{UCQ})$ can be converted to UCQ queries with inequality, to our knowledge, the complexity on equivalent query rewriting for UCQ with inequality is not yet known (some special cases are considered in [4]). Our results on SWS composition may shed light on the study of equivalent query rewriting.

For SWS composition synthesis we show the following. First, it is infeasible already for nonrecursive SWS's and mediators defined in terms of FO. Second, it remains undecidable for goal services in SWS(CQ, UCQ) even when either mediators or components are nonrecursive, but it becomes decidable when all of them are nonrecursive. Third, for SWS(PL, PL) composition synthesis involves arbitrary combinations of concatenation, intersection and complementation of SWS's, and is more intriguing than the rewriting problems studied for regular path queries in previous work (e.g., [8]). While we do not yet know whether it is decidable in general, we show that it is decidable when either goal SWS's or both mediators and components are nonrecursive.

Theorem 5.1: Composition synthesis $\operatorname{CP}(\mathcal{G}, \mathcal{M}, \mathcal{C})$ is

1. undecidable when $\mathcal{G}, \mathcal{C}$ are $\operatorname{SWS}(\mathrm{FO}, \mathrm{FO})$ and $\mathcal{M}$ is $\operatorname{MDT}(\mathrm{FO})$, even when $\mathcal{G}, \mathcal{M}, \mathcal{C}$ are all nonrecursive;

2. undecidable when $\mathcal{G}, \mathcal{C}$ are $\operatorname{SWS}(\mathrm{CQ}, \mathrm{UCQ})$ and $\mathcal{M}$ is $\operatorname{MDT}(\mathrm{UCQ})$, even when either $\mathcal{M}$ or $\mathcal{C}$ is nonrecursive;
3. in 2EXPSPACE when $\mathcal{G}, \mathcal{C}$ are $\operatorname{SWS}_{\mathrm{nr}}(\mathrm{CQ}, \mathrm{UCQ})$ and $\mathcal{M}$ is $\mathrm{MDT}_{\mathrm{nr}}(\mathrm{UCQ})$, i.e., when services and mediators are all nonrecursive;

4. decidable when $\mathcal{G}$ is $\mathrm{SWS}_{\mathrm{nr}}(\mathrm{PL}, \mathrm{PL}), \mathcal{C}$ is $\mathrm{SWS}(\mathrm{PL}, \mathrm{PL})$ and $\mathcal{M}$ is $\operatorname{MDT}(\mathrm{PL})$; and

5. decidable when $\mathcal{G}$ is $\operatorname{SWS}(\mathrm{PL}, \mathrm{PL}), \mathcal{C}$ is $\mathrm{SWS}_{\mathrm{nr}}(\mathrm{PL}, \mathrm{PL})$ and $\mathcal{M}$ is $\mathrm{MDT}_{\mathrm{nr}}(\mathrm{PL})$.

Proof. (1) The undecidability of $\mathrm{CP}\left(\mathrm{SWS}_{\mathrm{nr}}(\mathrm{FO}, \mathrm{FO})\right.$, $\left.\operatorname{MDT}_{\mathrm{nr}}(\mathrm{FO}), \mathrm{SWS}_{\mathrm{nr}}(\mathrm{FO}, \mathrm{FO})\right)$ is verified by reduction from the satisfiability problem for FO queries.

(2) When either mediators or component services are nonrecursive, the undecidability can already be established by reduction from the equivalence problem for SWS(CQ,UCQ), which is proved undecidable by Theorem 4.1.

(3) We first show that $\mathrm{CP}\left(\mathrm{SWS}_{\mathrm{nr}}(\mathrm{CQ}, \mathrm{UCQ}), \mathrm{MDT}_{\mathrm{nr}}(\mathrm{UCQ})\right.$, $\left.\mathrm{SWS}_{\mathrm{nr}}(\mathrm{CQ}, \mathrm{UCQ})\right)$ can be reduced to the problem for equivalent query rewriting using views for UCQ with $\neq$, in EXPTIME. We then show that the rewriting problem is decidable in EXPSPACE for UCQ queries with $\neq$, by establishing a small model property, i.e., if there exists an equivalent rewriting then there exists one bounded by EXPSPACE.

(4) We use the notion of $k$-prefix recognizable languages, i.e., languages for which membership is determined by the first $k$ symbols of the input sequence, for some $k \in \mathbb{N}$. We show that for each $\tau$ in $\operatorname{SWS}_{\mathrm{nr}}(\mathrm{PL}, \mathrm{PL})$ there is a $k$ such that $\tau$ defines a $k$-prefix recognizable language. Moreover, given $k$, there is a bound on the size of mediators defining $k$-prefix recognizable languages. Thus we can guess a mediator of bounded size and check equivalence with the goal SWS.

(5) Similarly, we show that an $\mathrm{MDT}_{\mathrm{nr}}(\mathrm{PL})$ mediator over component SWS's in $\mathrm{SWS}_{\mathrm{nr}}(\mathrm{PL}, \mathrm{PL})$ is only capable of defining $k$-prefix recognizable languages, for some $k \in \mathbb{N}$. Further, we show that if the language defined by the goal SWS $\tau$ is $k$-prefix recognizable, then we can put a bound on the value of $k$. If $\pi$ and $\tau$ are equivalent, $\tau$ must be $k$-prefix recognizable. This again yields a bound on the size of possible mediators equivalent to $\tau$, and thus decidability.

For SWS(CQ, UCQ), one might be tempted to consider finding a nonrecursive mediator $\pi$ over nonrecursive component SWS's for a recursive goal SWS $\tau$ such that $\pi \equiv \tau$. Indeed, there has been work on equivalence between recursive and nonrecursive datalog programs [10]. There is, however, a subtle difference between equivalence of SWS's and mediators, and equivalence on datalog queries. To see this, recall the runs of SWS's and mediators. Each time when an execution tree is expanded, i.e., whenever new leaf nodes are spawned, an input message in the sequence $\mathcal{I}$ is consumed, by SWS's and (component services in) mediators alike. In other words, the computation steps of an SWS or a mediator is bounded by the length of $\mathcal{I}$. Therefore, while the computation of a nonrecursive $\pi$ can be conducted in a fixed number of steps, the computation of a recursive $\tau$ may need an unbounded number of steps. Hence one can find a long enough sequence $\mathcal{I}$ of input messages such that different outputs are produced by $\pi$ and $\tau$ on $\mathcal{I}$. In other words, $\pi$ and $\tau$ are not equivalent on all input sequences. Indeed, in this setting the composition synthesis problem is reduced to composition synthesis of nonrecursive goal SWS's and nonrecursive mediators and components, i.e., case (3) above. This is what the proof of (5) does for SWS(PL, PL): only $k$-prefix recognizable goal services make sense in that 
context. In contrast, this is not an issue for the study of equivalence between datalog programs.

For the same reason, as indicated in the proof of (4), for a goal SWS $\tau$ in $\operatorname{SWS}_{\mathrm{nr}}(\mathrm{CQ}, \mathrm{UCQ})$, it suffices to consider only nonrecursive mediators and component SWS's, i.e., case (3). This is in contrast to containment of nonrecursive datalog programs in recursive datalog programs (e.g., [11]).

Special cases. Theorem 5.1 tells us that in general, composition synthesis for data-driven SWS's is beyond reach in the presence of recursion. Below we identify a decidable case for recursive data-driven services in SWS(CQ,UCQ), by leveraging a result on UC2RPQ queries established in [9].

A 2-way regular path query (2RPQ) $Q$ expresses a regular expression on a semistructured database $D$. The database is an edge-labeled graph, encoded by a collection of binary relations for edges, along with their inverse. On $D$ the query $Q$ computes a set $\left(d_{0}, d_{q}\right)$ of nodes such that $e_{1}\left(d_{0}, d_{1}\right), \ldots, e_{q}\left(d_{q-1}, d_{q}\right)$ is a path in $D$ and $e_{1}, \ldots, e_{q}$ is a word in the regular language of $Q$. Unions of conjunctions of 2RPQ's can be naturally defined, denoted by UC2RPQ's. It is shown [9] that containment of UC2RPQ's is decidable in 2ExPTIME. One can express a UC2RPQ in $\mathrm{SWS}(\mathrm{CQ}, \mathrm{UCQ})$. Let us denote by $\mathrm{SWS}(\mathrm{UC} 2 \mathrm{RPQ})$ the class of all SWS's in SWS(CQ,UCQ) that express UC2RPQ's, and by MDT(UC2RPQ) the class of all mediators in MDT(UCQ) that are equivalent to some SWS in SWS(UC2RPQ). We also denote by $\mathrm{SWS}_{\mathrm{nr}}\left(\mathrm{CQ}^{r}\right)$ the subclass of $\mathrm{SWS}_{\mathrm{nr}}(\mathrm{CQ}, \mathrm{UCQ})$ such that each SWS in the class expresses a CQ query (with two states). We show below that given a goal service $\tau$ in SWS(UC2RPQ) and a set $S$ of components in $\mathrm{SWS}_{\mathrm{nr}}\left(\mathrm{CQ}^{r}\right)$, it is decidable in 2EXPTIME to determine whether or not there exists a mediator $\pi$ in MDT(UC2RPQ) such that $\pi \equiv \tau$.

Corollary 5.2: The synthesis problem CP(SWS(UC2RPQ), $\left.\operatorname{MDT}(\mathrm{UC} 2 \mathrm{RPQ}), \mathrm{SWS}_{\mathrm{nr}}\left(\mathrm{CQ}^{r}\right)\right)$ is decidable in 2EXPTIME. $\quad \square$

Proof. One can verify that the composition synthesis problem is PTIME equivalent to the problem for equivalent query rewriting for UC2RPQ queries using CQ views. Further, using the maximally-contained rewriting algorithm developed for datalog [14] one can show that if an equivalent rewriting of a UC2RPQ query exists, then an equivalent UC2RPQ rewriting can be found in PTIME. From these and [9] the 2ExPTIME upper bound follows.

While the composition synthesis problem is open when goal and component services are in SWS(PL, PL) and mediator is in $\operatorname{MDT}(\mathrm{PL})$, we identify several decidable cases. First, the analysis is simplified when only disjunction is allowed in the synthesis queries of mediators. We denote this subclass of $\mathrm{MDT}(\mathrm{PL})$ by $\mathrm{MDT}(\mathrm{V})$. In this setting the problem becomes decidable in 3EXPSPACE when mediators, goal and component services are all recursive. Second, we can reduce the complexity if goal services are NFA's (2EXPSPACEcomplete) or DFAs (in EXPSPACE), as found in, e.g., the Roman model. Third, we restrict MDT(PL) such that each component services is invoked at most a fixed number of times in all transition rules combined, and the sizes of the synthesis functions are bounded. We refer to MDT(PL) with such a bound as $\mathrm{MDT}^{b}(\mathrm{PL})$. Then the composition synthesis problem is decidable in EXPSPACE, and it is PSPACE-complete if only nonrecursive component services are allowed.

Theorem 5.3:

1. $\mathrm{CP}(\mathrm{SWS}(\mathrm{PL}, \mathrm{PL}), \mathrm{MDT}(\vee), \mathrm{SWS}(\mathrm{PL}, \mathrm{PL}))$ is decidable in 3EXPSPACE;
2. $\mathrm{CP}(\mathrm{NFA}, \operatorname{MDT}(\mathrm{V})$, SWS(PL, PL)) is 2EXPSPACEcomplete, while $\mathrm{CP}(\mathrm{DFA}, \mathrm{MDT}(\mathrm{V})$, $\mathrm{SWS}(\mathrm{PL}, \mathrm{PL}))$ is in EXPSPACE;

3. $\mathrm{CP}\left(\mathrm{SWS}(\mathrm{PL}, \mathrm{PL}), \mathrm{MDT}^{b}(\mathrm{PL}), \mathrm{SWS}(\mathrm{PL}, \mathrm{PL})\right)$ is in EXPSPACE; CP(SWS(PL, PL), $\left.\operatorname{MDT}^{b}(\mathrm{PL}), \mathrm{SWS}_{\mathrm{nr}}(\mathrm{PL}, \mathrm{PL})\right)$ is PSPACE-complete.

Proof. (1) Every SWS(PL,PL) is translated in exponential time to an equivalent NFA. The proof then employs the 2EXPSPACE NFA rewriting algorithm of [8], taking into account the subtle interplay between a mediator and the SWS's it calls. That is, the corresponding NFA's should stop processing the input the first time a final state is encountered. The overall algorithm is therefore in 3EXPSPACE.

(2) When goal services can be expressed as an NFA, and mediators are in $\operatorname{MDT}(\mathrm{V})$ we show that composition synthesis is PTIME-equivalent to the rewriting problem of [8] for NFA. From these and the complexity bounds of [8] follows the result for an NFA goal. We show that the bound can be lowered to EXPSPACE for a DFA goal.

(3) For $\mathrm{MDT}^{b}(\mathrm{PL})$, composition synthesis has a small model property: if a mediator in $\mathrm{MDT}^{b}(\mathrm{PL})$ exists, then there must be one of which the size is bounded by a polynomial in the size of the goal SWS and component SWS's. Further, testing equivalence of an SWS and such a mediator can be done in EXPSPACE in general, and in PSPACE when the component SWS's are nonrecursive. From this the upper bounds of (3) follow. The PSPACE lower bound is by a reduction from nonemptiness analysis of SWS(PL,PL) (Theorem 4.1 (3)).

It should be remarked that SWS composition is quite different from service composition studied for the Roman model [6]. In contrast to the Roman model that allows interleaving of executions of component services, SWS composition requires that component services run to completion in order to synthesize their actions. The difference in the semantics leads to different complexity bounds on the synthesis: it is EXPTIME-complete for the Roman model $[6,24]$, whereas it is 2EXPSPACE-hard for SWS(PL,PL) composition.

\section{Conclusion}

We have proposed a notion of synthesized Web services to uniformly characterize FSA and transducer abstractions of Web services. Moreover, SWS's decouple unnecessary temporal dependencies imposed by FSA abstractions, and facilitate deterministic synthesis of service actions. We have established matching lower and upper bounds on the nonemptiness, validation and equivalence problems associated with various classes of SWS's. We have also provided complexity bounds on their composition synthesis.

The main results are summarized in Tables 1 and 2. Consistent with the findings of $[2,5,6,12,13,16,24,29]$, the decision and composition synthesis problems are either undecidable or highly intractable for recursively defined services. In light of these negative results we have identified decidable cases and special cases with lower complexity bounds. Moreover, our proof techniques, e.g., finding service composition via query rewriting using views, may help develop practical heuristic algorithms in certain application domains.

There is naturally much more to be done. First, the composition synthesis problem is open when goal and component services are in SWS(PL, PL) and mediators are in $\operatorname{MDT}(\mathrm{PL})$. This is, however, nontrivial. In fact the proofs 


\begin{tabular}{|c|c|c|c|}
\hline \multicolumn{3}{|c|}{$\mathrm{CP}(\mathcal{G}, \mathcal{M}, \mathcal{C})$} & \multirow[t]{2}{*}{ Complexity } \\
\hline $\mathcal{G}$ : goal service & $\mathcal{M}:$ mediator & $\mathcal{C}:$ component & \\
\hline SWS(FO,FO) & $\mathrm{MDT}(\mathrm{FO})$ & SWS(FO,FO) & undecidable \\
\hline $\mathrm{SWS}_{\mathrm{nr}}(\mathrm{FO}, \mathrm{FO})$ & $\mathrm{MDT}_{\mathrm{nr}}(\mathrm{FO})$ & $\mathrm{SWS}_{\mathrm{nr}}(\mathrm{FO}, \mathrm{FO})$ & undecidable \\
\hline 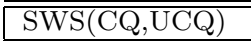 & $\overline{\mathrm{MDT}(\mathrm{UCQ})}$ & 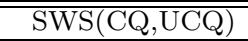 & undecidable \\
\hline SWS( & $\operatorname{MDT}(\mathrm{UCQ})$ & $\mathrm{SWS}_{\mathrm{nr}}(\mathrm{CQ}$ & idable \\
\hline SWS(CQ,UCQ) & $\mathrm{MDT}_{\mathrm{nr}}(\mathrm{UCQ})$ & SWS(CQ,UCQ) & undecidable \\
\hline $\mathrm{SWS}_{\mathrm{nr}}(\mathrm{CQ}, \mathrm{UCQ})$ & $\mathrm{MDT}_{\mathrm{nr}}(\mathrm{UCQ})$ & $\mathrm{SWS}_{\mathrm{nr}}(\mathrm{CQ}, \mathrm{UCQ})$ & 2EXPSPACE \\
\hline SWS & MDT(PL) & SWS(PL,PL) & le \\
\hline SW & $\mathrm{MDT}_{\mathrm{nr}}(\mathrm{PL})$ & SWS & idable \\
\hline \multicolumn{3}{|c|}{ Special cases } & \\
\hline SWS & 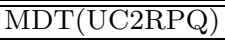 & $\left(\mathrm{CQ}^{r}\right)$ & $2 \mathrm{EXI}$ \\
\hline SWS(PL,PL) & $\operatorname{MDT}(\mathrm{V})$ & SWS(PL,PL) & 3EXPSPACE \\
\hline NFA & $\operatorname{MDT}(\mathrm{V})$ & SWS(PL, PL) & 2EXPSPACE \\
\hline DFA & $\operatorname{MDT}(\vee)$ & SWS(PL, PL) & EXPSPACE \\
\hline SWS & $\mathrm{MDT}^{b}(\mathrm{PL})$ & SWS(PL, PL) & EXPSPACE \\
\hline SWS(PL, PL) & $\operatorname{MDT}^{b}(\mathrm{PL})$ & $\mathrm{SWS}_{\mathrm{nr}}(\mathrm{PL}, \mathrm{PL})$ & PSPACE \\
\hline
\end{tabular}

Table 2: Complexity of composition synthesis

for its special cases (Theorem $5.1(4,5)$ ) are already nonelementary. Second, in several decidable composition cases, we have only provided upper bounds, for which the exact complexity remains unknown. Third, the undecidability and highly intractable results suggest that we identify practical restrictions that allow decidable and better still, tractable static analyses and composition synthesis. Fourth, a practical topic for future work is to extend SWS's by incorporating aggregation and a cost model into action synthesis to find, e.g., a travel package with minimum total cost when airfare, hotel and other components are all taken together. While aggregation on composed services is certainly needed in practice, we are not aware of any formal study of this issue. Finally, we also plan to investigate for SWS's the verification problems and restrictions studied in [12, 13], as well as the impact of lossy channel [13] and global constraints [5, 15] on static analyses and composition synthesis of SWS's.

Acknowledgments. Wouter Gelade is Research Assistant of the Fund for Scientific Research - Flanders (Belgium). Wenfei Fan is supported in part by EPSRC GR/S63205/01, GR/T27433/01 and EP/E029213/1. Floris Geerts is supported in part by EPSRC EP/E029213/1.

\section{References}

[1] S. Abiteboul, R. Hull, and V. Vianu. Foundations of Databases. Addison-Wesley, 1995.

[2] S. Abiteboul, V. Vianu, B. S. Fordham, and Y. Yesha. Relational transducers for electronic commerce. JCSS, 61(2):236-269, 2000.

[3] F. N. Afrati, M. Gergatsoulis, and T. G. Kavalieros. Answering queries using materialized views with disjunctions. In ICDT, 1999.

[4] F. N. Afrati, C. Li, and P. Mitra. Rewriting queries using views in the presence of arithmetic comparisons. Theor. Comput. Sci., 368(1-2):88-123, 2006.

[5] D. Berardi, D. Calvanese, G. D. Giacomo, R. Hull, and M. Mecella. Automatic composition of transition-based semantic web services with messaging. In $V L D B, 2005$.

[6] D. Berardi, D. Calvanese, G. D. Giacomo, M. Lenzerini, and M. Mecella. Automatic service composition based on behavioral descriptions. Int. J. Cooperative Inf. Syst., 14(4):333-376, 2005.

[7] Business Process Execution Language for Web Services version 1.1 (BEPL4WS), 2004. http://www.ibm.com/ developerworks/library/specification/ws-bpel/.

[8] D. Calvanese, G. D. Giacomo, M. Lenzerini, and M. Y. Vardi. Rewriting of regular expressions and regular path queries. JCSS, 64(3):443-465, 2002.

[9] D. Calvanese, G. D. Giacomo, and M. Y. Vardi. Decidable containment of recursive queries. TCS, 336(1):33$56,2005$.

[10] S. Chaudhuri and M. Y. Vardi. On the equivalence of recursive and nonrecursive datalog programs. JCSS, 54(1):61-78, 1997.

[11] S. S. Cosmadakis and P. C. Kanellakis. Parallel evaluation of recursive rule queries. In PODS, 1986.

[12] A. Deutsch, L. Sui, and V. Vianu. Specification and verification of data-driven Web applications. JCSS, 73(3):442-474, 2007.

[13] A. Deutsch, L. Sui, V. Vianu, and D. Zhou. Verification of communicating data-driven Web services. In PODS, 2006.

[14] O. M. Duschka and M. R. Genesereth. Answering recursive queries using views. In PODS, 1997.

[15] X. Fu, T. Bultan, and J. Su. Analysis of interacting BPEL Web services. In $W W W, 2004$.

[16] X. Fu, T. Bultan, and J. Su. Conversation protocols: a formalism for specification and verification of reactive electronic services. TCS, 328(1-2):19-37, 2004.

[17] M. Garey and D. Johnson. Computers and intractability: A guide to the theory of NP-completeness. W. H. Freeman and Company, 1979.

[18] C. E. Gerede, R. Hull, O. H. Ibarra, and J. Su. Automated composition of e-services: lookaheads. In $I C$ SOC, 2004.

[19] G. Gottlob and C. Papadimitriou. On the complexity of single rule datalog queries. Inf. Comput., 183(1):104$122,2003$.

[20] A. Y. Halevy. Theory of answering queries using views. SIGMOD Record, 29(4), 2001.

[21] R. Hull and J. Su. Tools for composite web services: a short overview. SIGMOD Record, 34(1):5-12, 2005.

[22] A. Klug. On conjunctive queries containing inequalities. J. ACM, 35(1):146-160, 1988.

[23] A. Levy, A. O. Mendelzon, Y. Sagiv, and D. Srivastava. Answering queries using views. In PODS, 1995.

[24] A. Muscholl and I. Walukiewicz. A lower bound on Web services composition. In FoSSaCS, 2007.

[25] OWL-S: Semantic Markup for Web Services, 2004. http://www.w3.org/Submission/OWL-S/.

[26] M. Pistore, P. Traverso, P. Bertoli, and A. Marconi. Automated synthesis of composite BPEL4WS web services. In $I S W C, 2005$.

[27] Semantic Web Services Framework (SWSF) Version 1.1, 2005. http://www.daml.org/services/swsf/1.1/.

[28] M. Spielmann. Abstract State Machines: Verification Problems and Complexity. PhD thesis, RWTH , 2000.

[29] M. Spielmann. Verification of relational transducers for electronic commerce. JCSS, 66(1):40-65, 2003.

[30] Web Services Conversation Language (WSCL) 1.0, 2002. http://www.w3.org/TR/wscl10/.

[31] Web Services Description Language (WSDL) 1.1, 2001. http://www.w3.org/TR/wsdl.

[32] S. Yu. Regular languages. In Handbook of Formal Languages, volume 1. Springer, 1996. 